\title{
FLORA Y VEGETACION DEL SALAR DE ASCOTAN, ANDES DEL NORTE DE CHILE
}

\section{FLORA AND VEGETATION OF ASCOTAN SALTMARSH, NORTHERN CHILEAN ANDES}

\author{
Sebastián Teillier ${ }^{1} \&$ Pablo Becerra ${ }^{2}$ \\ ${ }^{1}$ Escuela de Ecología y Paisajismo, Facultad de Arquitectura, Universidad Central, Santa Isabel 1186, \\ Santiago, Chile. E-mail: steillier@ chlorischile.cl \\ ${ }^{2}$ Departamento de Ciencias Ecológicas, Facultad de Ciencias, Universidad de Chile, Casilla 653, Santiago, \\ Chile.
}

\section{RESUMEN}

En este trabajo se caracterizan la flora y la vegetación del salar de Ascotán, ubicado en los Andes de la Región de Antofagasta (II), Chile. La flora del salar, compuesta por 21 especies, es completamente diferente de la flora aledaña a él. También difieren los espectros de formas de vida del salar, dominado por hemicriptófitas (hierbas perennes), con la vegetación que lo rodea, dominada por nanofanerófitas (arbustos). En el salar se encontraron dos unidades de vegetación diferenciadas a partir por la composición de especies. Ambas unidades difieren, tanto en su flora como en sus espectros biológicos. Una unidad está dominada por hemicriptófitas y caméfitas, mientras que en la otra dominan exclusivamente las hemicriptófitas; las caméfitas y las geófitas poseen menor importancia. Factores edáficos probablemente pueden explicar las diferencias vegetacionales entre el salar y sus alrededores, como la presencia de dos unidades vegetacionales dentro del salar.

Palabras claves: Análisis de conglomerados, fitosociología, formas de vida, halófitas, humedales andinos.

\begin{abstract}
In this paper we characterise the flora and vegetation of Ascotan saltmarsh, located in the Andes of northern Chile. The saltmarsh flora, comprising 21 species, is completely different from the flora of surrounding slopes, differing not only in species composition but also in lifeform spectrum. The saltmarsh is dominated by hemicryptophytes, whereas nanophanerophytes predominate on the slopes. A cluster analysis distinguished two vegetation types within the saltmarsh, differing in species composition and in representation of chamaephytes, geophytes and hemicriptophytes. Substrate variation probably accounts for differences between the saltmarsh vegetation and its surrounding sites, as well as the existence of two vegetation types within the salar.
\end{abstract}

Keywords: Andean marshes, cluster analysis, phytosociology, halophytes, life forms. 


\section{INTRODUCCION}

Los Andes del Norte Grande de Chile presentan cuencas cerradas, situadas entre los diferentes cordones que forman la cordillera, que en la actualidad presentan depósitos salinos denominados comúnmente "salares" (Brüggen 1950). Estos habrían resultado de la desecación de lagos formados como consecuencia del derretimiento de los glaciares, producido entre 15.000 y 10.000 años A.P. (Ochsenius 1986; Messerli et al. 1993). En la actualidad, esos depósitos salinos no presentan grandes variaciones geomorfológicas ni geológicas internas. Se encuentran en cuencas planas casi sin gradientes topográficos y están formados por una fracción líquida correspondiente a salmueras, una fracción salina representada por la depositación de diferentes sales transportadas en solución a la cuenca, y una fracción sólida constituida por distintos niveles de arena, limo y arcilla (Vila 1975). Prácticamente a ninguno de los salares del norte de Chile (excepto al de Tara) confluyen cauces superficiales permanentes de importancia, y la gran mayoría son alimentados sólo por las lluvias y aguas subterráneas, suficientes como para formar pequeñas lagunas, con un nivel freático inestable (Vila 1975). El sistema hidrológico de los salares está formado por surgencias, canales y lagunas. La salinidad varía desde niveles muy bajos en los sitios de surgencia, hasta máximos en el interior de las lagunas o riberas alejadas de la surgencia. Esta variación de la salinidad del agua se corresponde con cambios en la flora acuática vascular y algal y, también, en la fauna.

Las plantas adaptadas para vivir en condiciones de alta salinidad se conocen como halófitas, y generalmente forman comunidades particulares, diferentes de hábitats menos salinos (Braun-Blanquet 1979). En los salares andinos, esto debiera expresarse en fuertes diferencias de flora entre éstos y los hábitats aledaños. Además, la variabilidad edáfica, especialmente de salinidad y humedad dentro de los salares, también debiera determinar una importante variabilidad florística al interior de ellos.

La forma de vida es un carácter morfológico que otorga a las plantas una mayor o menor adecuación para sobrevivir y reproducirse bajo determinadas características ambientales, es- pecialmente de clima (Raunkiaer 1934; BraunBlanquet 1979). Por esto, la forma de vida puede ser importante en la variación de especies entre hábitats (Cody 1986; Tilman 1988; Danin \& Orshan 1990). Sin embargo, se desconoce si en los ambientes salinos de desierto, la forma de vida es un carácter importante en la estructuración de la composición de especies halófitas, pudiendo influir así en la gran variación florística entre un salar y los hábitats aledaños y al interior de un salar.

Todos estos aspectos son aún poco conocidos en los salares andinos. Estas comunidades se han estudiado relativamente poco, tanto en Chile (e.g. Villagrán et al.1983; Gajardo 1994; Teillier 1998), como en países limítrofes (e.g. Cabrera 1957; Navarro 1993), en contraste a los más numerosos estudios de flora y vegetación de otras comunidades del norte de Chile (Arroyo et al. 1982; Villagrán et al. 1982; Arroyo et al. 1988; Rundel et al. 1996; Armesto et al. 1993; Gajardo 1994; Teillier 1998, 2000; Luebert \& Gajardo 2000; Becerra \& Faúndez 2001).

Este trabajo caracteriza la flora y la vegetación del salar de Ascotán, ubicado en la Cordillera de los Andes, Región de Antofagasta (II), Chile. En particular, se compara la flora del salar con la de los hábitats circundantes y se evalúa la existencia de variaciones de flora al interior del salar, determinante de diferentes unidades de vegetación. Además, se caracteriza el espectro de formas de vida del salar y hábitats aledaños y de las unidades vegetacionales presentes al interior del salar.

\section{MATERIAL Y METODOS}

\section{Area de estudio}

El trabajo se llevó a cabo en el salar de Ascotán, situado en la Cordillera de los Andes de la Región de Antofagasta (II), al nororiente de la ciudad de Calama (21 ${ }^{\circ} 29^{\prime} \mathrm{S}, 68^{\circ} 19^{\prime} \mathrm{W}$ ) a una elevación de 3.800 msnm (Fig. 1). El salar se ubica en una gran cuenca orientada de $\mathrm{N}$ a $\mathrm{S}$ que está limitada, al oeste, por el Cordón Occidental de la Cordillera de los Andes, con los cerros del Azufre (5.846 m), Carasilla (5.040 m) y Polapi (5.940 m); hacia el este, por el Cordón Oriental, con los 
cerros Ascotán-Jardín (5478 m) y Araral (5688 $\mathrm{m})$; hacia el sur, la cuenca se cierra con los faldeos asociados a los cerros Ascotán y Azufre, $y$, hacia el norte, limita con el sector de las serranías que la separan del salar de Carcote.

El clima del área de estudio corresponde a uno de tendencia tropical (Di Castri \& Hajek 1976) y presenta un importante grado de aridez, ya que las precipitaciones, de acuerdo con los datos de la estación climática más cercana (Ollagüe), alcanzan en promedio sólo a unos 80 $\mathrm{mm}$. Las precipitaciones se concentran principalmente en el período correspondiente al verano, aunque también se pueden registrar precipitaciones sólidas (nieve y granizo) durante el período invernal.

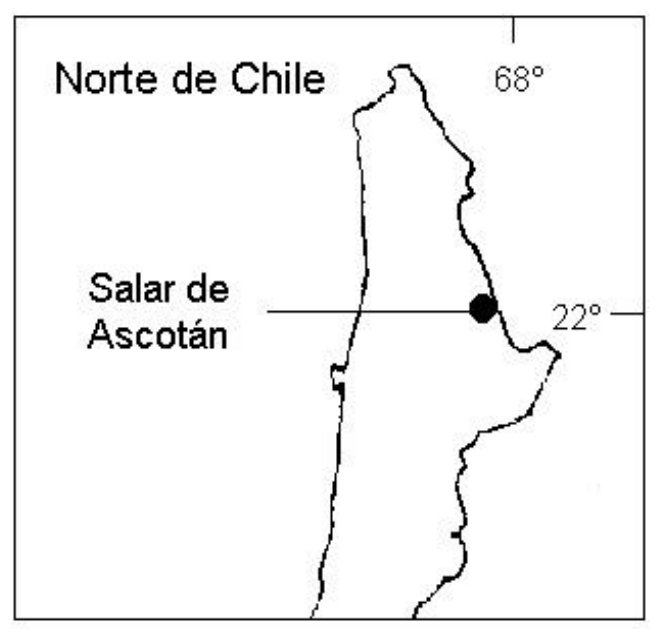

FiguRA 1. Ubicación geográfica del salar de Ascotán en el norte de Chile.

\section{MuestReO Y ANÁLISIS}

El área del estudio fue visitada varias veces entre los años 1993 y 1998. El registro de la flora que forma la vegetación del salar (flora azonal) y de las laderas aledañas al salar (flora zonal) se estableció mediante rastreos intensivos en cada sector. La variación florística al interior del salar se evaluó a partir de 46 parcelas de $4 \mathrm{~m}^{2}$, correspondiente al área mínima de muestreo para este tipo de comunidad (MüllerDombois \& Ellenberg 1974). Para cada levantamiento se registró la composición de las especies de plantas vasculares y el porcentaje de cobertura para cada una por medio de estimación visual. Las parcelas fueron distribuidas en ocho parches de vegetación, cada uno asociado a una laguna distinta, con un número variable de inventarios por parche (4 a 14).

Usando estas parcelas, se realizó una análisis de conglomerados mediante un algoritmo "Complete Linkage", y se utilizó el índice de disimilitud como medida de comparación de la composición de especies. La abundancia de las especies, para cada unidad de vegetación resultante del análisis de conglomerados, se caracterizó mediante parámetros como la frecuencia relativa y la cobertura relativa, los que varían entre 0 y 100. Además, se calculó un valor de importancia, correspondiente a la suma de la frecuencia y la cobertura relativa para cada especie. Este valor, por lo tanto, varía entre 0 y 200.

Para caracterizar los espectros de formas de vida de las laderas aledañas al salar, del salar y de las unidades vegetacionales de éste, se asignó a cada especie registrada una forma de vida según Raunkiaer (1934). Los espectros del salar y flora zonal se determinaron sólo con el número y porcentaje de especies por forma de vida, mientras que los espectros de las unidades de vegetación se establecieron, además, por la cobertura total de las especies de cada forma de vida.

Los análisis de comparación de la composición de especies y los espectros de las formas de vida se realizaron considerando sólo a las especies de plantas terrestres, aunque también se entrega información sobre la flora acuática vascular de las lagunas y las vertientes del salar.

La nomenclatura de las especies sigue a Marticorena y Quezada (1985) con correcciones sugeridas por el primero de estos autores. Los ejemplares colectados se depositaron en los Herbarios del Museo Nacional de Historia Natural (SGO) y de la Universidad de Concepción (CONC) (ver Teillier 2000).

\section{RESULTADOS}

Para el salar de Ascotán se registraron 21 especies de plantas vasculares, 18 terrestres y 3 acuáticas, pertenecientes a 12 familias y 17 géneros. Las familias mejor representadas fueron Poaceae (Gramineae) y Asteraceae (Compositae) (Tabla I). La flora zonal de las laderas aledañas al salar estuvo 
formada por 17 especies, correspondiente principalmente a las familias Asteraceae y Verbenaceae (Tabla I). En relación con la composición de las floras, no hubo especies en común entre el salar y las laderas aledañas.

El análisis de conglomerados distinguió principalmente dos agrupaciones en el salar, las cuales presentaron un $65 \%$ de especies diferentes (Fig. 2). Una primera unidad (A), presente en todas las lagunas del salar, estuvo dominada, en términos de frecuencia, por Puccinellia frigida (Phil.) I.M.Johnst., Scirpus atacamensis (Phil.) Boeck y Sarcocornia pulvinata (R.E.Fries) A.J.Scott, y en términos de cobertura, por S. atacamensis y S. pulvinata (Tabla II). Al considerar el valor de importancia, la unidad A estuvo dominada por S. atacamensis y P. frigida. La segunda unidad (B) estuvo dominada en frecuencia por S. atacamensis, Festuca hypsophila Phil.y Deyeuxia eminens J.Presl, y en cobertura por Juncus arcticus Willd., F. hypsophila y D. eminens. Al considerar el valor de importancia, la unidad B estuvo dominada por $S$. atacamensis y Festuca deserticola Phil. (Tabla II).

TABLA I. Composición y formas de vida de la flora del salar de Ascotán y de la vegetación zonal aledaña.

\begin{tabular}{|c|c|c|}
\hline Especie & Familia & Forma de vida \\
\hline $\begin{array}{l}\text { FLORA DEL SALAR } \\
\text { Arenaria rivularis } \text { Phil. } \\
\text { Baccharis acaulis (Wedd. ex R.E.Fries.) Cabrera } \\
\text { Baccharis juncea (Lehm.) Desf. } \\
\text { Carex maritima Gunnerus. } \\
\text { Deyeuxia eminens J.Presl } \\
\text { Distichlis humilis } \text { Phil. } \\
\text { Distichlis spicata (L.) Greene } \\
\text { Festuca deserticola } \text { Phil. } \\
\text { Festuca hypsophila } \text { Phil. } \\
\text { Lobelia oligophylla } \text { (Wedd.)Lammers } \\
\text { Juncus arcticus Willd. } \\
\text { Lilaeopsis macloviana (Gand.) A.W.Hill } \\
\text { Lycium humile Phil. } \\
\text { Potamogeton strictus } \text { Phil. } \\
\text { Puccinellia frigida (Phil.) I.M.Johnst. } \\
\text { Ruppia filifolia (Phil.) Skottsb. } \\
\text { Sarcocornia pulvinata (R.E.Fries) A.J.Scott } \\
\text { Scirpus americanus } \text { Pers. } \\
\text { Scirpus atacamensis (Phil.) Boeck } \\
\text { Triglochin concinna } \text { Burtt Davy } \\
\text { Werneria incisa } \text { Phil. }\end{array}$ & $\begin{array}{c}\text { Caryophyllaceae } \\
\text { Asteraceae } \\
\text { Asteraceae } \\
\text { Cyperaceae } \\
\text { Poaceae } \\
\text { Poaceae } \\
\text { Poaceae } \\
\text { Poaceae } \\
\text { Poaceae } \\
\text { Campanulaceae } \\
\text { Juncaceae } \\
\text { Apiaceae } \\
\text { Solanaceae } \\
\text { Potamogetonaceae } \\
\text { Poaceae } \\
\text { Ruppiaceae } \\
\text { Chenopodiaceae } \\
\text { Cyperaceae } \\
\text { Cyperaceae } \\
\text { Juncaginaceae } \\
\text { Asteraceae }\end{array}$ & $\begin{array}{c}\text { Hemicriptófita } \\
\text { Caméfita } \\
\text { Nanofanerófita } \\
\text { Hemicriptófita } \\
\text { Hemicriptófita } \\
\text { Hemicriptófita } \\
\text { Hemicriptófita } \\
\text { Hemicriptófita } \\
\text { Hemicriptófita } \\
\text { Hemicriptófita } \\
\text { Geófita } \\
\text { Hidrófita } \\
\text { Caméfita } \\
\text { Hidrófita } \\
\text { Hemicriptófita } \\
\text { Hidrófita } \\
\text { Caméfita } \\
\text { Geófita } \\
\text { Caméfita } \\
\text { Hemicriptófita } \\
\text { Caméfita }\end{array}$ \\
\hline $\begin{array}{l}\text { FLORA ZONAL (laderas) } \\
\text { Acantholippia punensis Botta } \\
\text { Adesmia erinacea } \text { Phil. } \\
\text { Adesmia horrida Gillies ex Hook et Arn. } \\
\text { Atriplex imbricata (Moq.) Dietr. } \\
\text { Baccharis boliviensis (Wedd.) Cabrera } \\
\text { Baccharis tola } \text { Phil. } \\
\text { Chuquiraga atacamensis Kuntze } \\
\text { Ephedra breana } \text { Phil. } \\
\text { Euphorbia amandi } \text { Oudejans } \\
\text { Fabiana denudata Miers } \\
\text { Hoffmannseggia doelli } \text { Phil. } \\
\text { Junellia seriphioides (Gillies et Hook.) Moldenke } \\
\text { Lampaya medicinalis } \text { Phil. } \\
\text { Senecio rosmarinus } \text { Phil. } \\
\text { Stipa frigida } \text { Phil. } \\
\text { Stipa venusta } \text { Hauman } \\
\text { Tarasa } \text { sp. }\end{array}$ & $\begin{array}{c}\text { Verbenaceae } \\
\text { Papilionaceae } \\
\text { Papilionaceae } \\
\text { Chenopodiaceae } \\
\text { Asteraceae } \\
\text { Asteraceae } \\
\text { Asteraceae } \\
\text { Ephedraceae } \\
\text { Euphorbiaceae } \\
\text { Solanaceae } \\
\text { Caesalpiniaceae } \\
\text { Verbenaceae } \\
\text { Verbenaceae } \\
\text { Asteraceae } \\
\text { Poaceae } \\
\text { Poaceae } \\
\text { Malvaceae } \\
\end{array}$ & $\begin{array}{c}\text { Nanofanerófita } \\
\text { Caméfita } \\
\text { Nanofanerófita } \\
\text { Nanofanerófita } \\
\text { Nanofanerófita } \\
\text { Nanofanerófita } \\
\text { Nanofanerófita } \\
\text { Nanofanerófita } \\
\text { Terófita } \\
\text { Nanofanerófita } \\
\text { Geófita } \\
\text { Nanofanerófita } \\
\text { Nanofanerófita } \\
\text { Nanofanerófita } \\
\text { Hemicriptófita } \\
\text { Hemicriptófita } \\
\text { Terófita } \\
\end{array}$ \\
\hline
\end{tabular}


Gayana Bot. 60(2), 2003

TABLA II. Abundancia de las especies terrestres por unidad de vegetación: se muestran la frecuencia (\%), la cobertura relativa (\%) y el valor de importancia (V.I.).

\begin{tabular}{lrcrrrr}
\hline \multirow{2}{*}{ Especies } & \multicolumn{3}{c}{ Unidad A } & \multicolumn{3}{c}{ Unidad B* } \\
\cline { 2 - 7 } & $\begin{array}{c}\text { Frec. } \\
\text { relativa }\end{array}$ & $\begin{array}{c}\text { Cob. } \\
\text { relativa }\end{array}$ & V. I. & $\begin{array}{c}\text { Frec. } \\
\text { relativa }\end{array}$ & $\begin{array}{c}\text { Cob. } \\
\text { relativa }\end{array}$ & V. I. \\
\hline Sarcocornia pulvinata & 14,2 & 15,6 & 29,7 & 0,0 & 0,0 & 0,0 \\
Baccharis acaulis & 6,3 & 4,7 & 11,0 & 0,0 & 0,0 & 0,0 \\
Arenaria rivularis & 6,3 & 1,5 & 7,8 & 0,0 & 0,0 & 0,0 \\
Werneria incisa & 4,7 & 7,8 & 12,5 & 0,0 & 0,0 & 0,0 \\
Baccharis juncea & 0,8 & 2,5 & 3,3 & 0,0 & 0,0 & 0,0 \\
Distichlis spicata & 0,8 & 2,5 & 3,3 & 0,0 & 0,0 & 0,0 \\
Puccinellia frigida & 24,4 & 11,9 & 36,3 & 6,7 & 2,3 & 9,0 \\
Distichlis humilis & 8,7 & 5,0 & 13,7 & 2,2 & 1,1 & 3,3 \\
Festuca deserticola & 0,0 & 0,0 & 0,0 & 15,6 & 12,1 & 27,7 \\
Carex maritima & 0,8 & 0,6 & 1,3 & 15,6 & 7,1 & 22,6 \\
Triglochin concinna & 6,3 & 1,7 & 8,1 & 13,3 & 2,7 & 16,0 \\
Festuca hypsophila & 3,9 & 3,9 & 7,8 & 6,7 & 17,6 & 24,3 \\
Deyeuxia eminens & 1,6 & 3,0 & 4,6 & 6,7 & 16,7 & 23,3 \\
Scirpus americanus & 0,0 & 0,0 & 0,0 & 2,2 & 0,1 & 2,3 \\
Juncus arcticus & 2,4 & 4,2 & 6,5 & 2,2 & 7,7 & 9,9 \\
Lycium humile & 3,2 & 3,9 & 7,1 & 2,2 & 0,1 & 2,3 \\
Scirpus atacamensis & 15,0 & 31,3 & 46,2 & 15,6 & 31,0 & 46,5 \\
\hline Se enconto sóy & & & & & &
\end{tabular}

* Se encontró sólo en la laguna de Palpana al interior del salar.

La flora terrestre del salar y la flora zonal de la ladera presentaron distintos espectros de forma de vida (Tabla III). En la flora del salar (azonal), predominaron las hemicriptófitas y no hubo terófitas. En la flora zonal, en tanto, hubo una mayor predominancia de especies nanofanerófitas, mientras que las hemicriptófitas presentaron igual importancia que las terófitas.

A pesar de la semejanza de los espectros de formas de vida, las dos unidades vegetacionales identificadas en el salar difirieron en la cobertura relativa de formas de vida (Tabla IV). El espectro de formas de vida de la unidad " A" estuvo dominado por las hemicriptófitas, seguido por las caméfitas, siendo sus porcentajes mucho mayores que los de las otras formas de vida. Sin embargo, si se considera la cobertura relativa de las formas de vida, predominaron las caméfitas, seguidas por las hemicriptófitas, mientras que las nanofanerófitas y las geófitas siguen presentando una menor importancia relativa. El espectro de riqueza de especies de la unidad B estuvo dominado ampliamente por las hemicriptófitas, siendo el resto aportado en partes iguales por caméfitas y geófitas. El espectro de cobertura de formas de vida fue similar al anterior, pero en este caso las caméfitas superaron a las geófitas y las hemicriptófitas siguieron siendo las dominantes. Luego, al considerar los espectros de riqueza por forma de vida, las unidades tuvieron cierta similitud, aunque en la unidad " $A$ " las caméfitas fueron relativamente más importantes y en la " $B$ ", no hay nanofanerófitas. Sin embargo, al considerar la cobertura por forma de vida, las unidades difirieron en mayor medida, por cuanto mientras la unidad " $A$ ", está dominada por caméfitas, la unidad " $\mathrm{B}$ " lo está por hemi-criptófitas. 
Flora y vegetación del salar de Ascotán: Teillier, S. \& P. Becerra

TABLA III. Espectros de formas de vida de la flora terrestre zonal y del salar de Ascotán.

\begin{tabular}{lcccc}
\hline Forma de vida & \multicolumn{2}{c}{ Azonal (salar) } & \multicolumn{2}{c}{ Zonal (laderas) } \\
\cline { 2 - 5 } & $\mathrm{N}^{\circ}$ & $\%$ & 1 & $\mathrm{~N}^{\circ}$ \\
\hline Caméfita & 4 & 19,1 & 2 & 5,9 \\
Hemicriptófita & 11 & 52,4 & 1 & 11,8 \\
Geófita & 2 & 9,5 & 11 & 5,9 \\
Nanofanerófita & 1 & 4,8 & 2 & 64,7 \\
Terófita & 0 & 0,0 & 0 & 11,8 \\
Hidrófita & 3 & 14,3 & & 0,0 \\
\hline
\end{tabular}

TABLA IV. Espectros de formas de vida de las unidades de vegetación del salar de Ascotán.

\begin{tabular}{lcccc}
\hline Forma de vida & \multicolumn{2}{c}{ Unidad A } & \multicolumn{2}{c}{ Unidad B } \\
\cline { 2 - 5 } & Riqueza (\%) & Cobertura (\%) & Riqueza (\%) & Cobertura (\%) \\
\hline Caméfita & 31,3 & 63,2 & 16,7 & 31,1 \\
Hemicriptófita & 56,3 & 30,2 & 66,7 & 61,1 \\
Geófita & 6,3 & 4,2 & 16,7 & 7,8 \\
Nanofanerófita & 6,3 & 2,5 & 0,0 & 0,0 \\
\hline
\end{tabular}

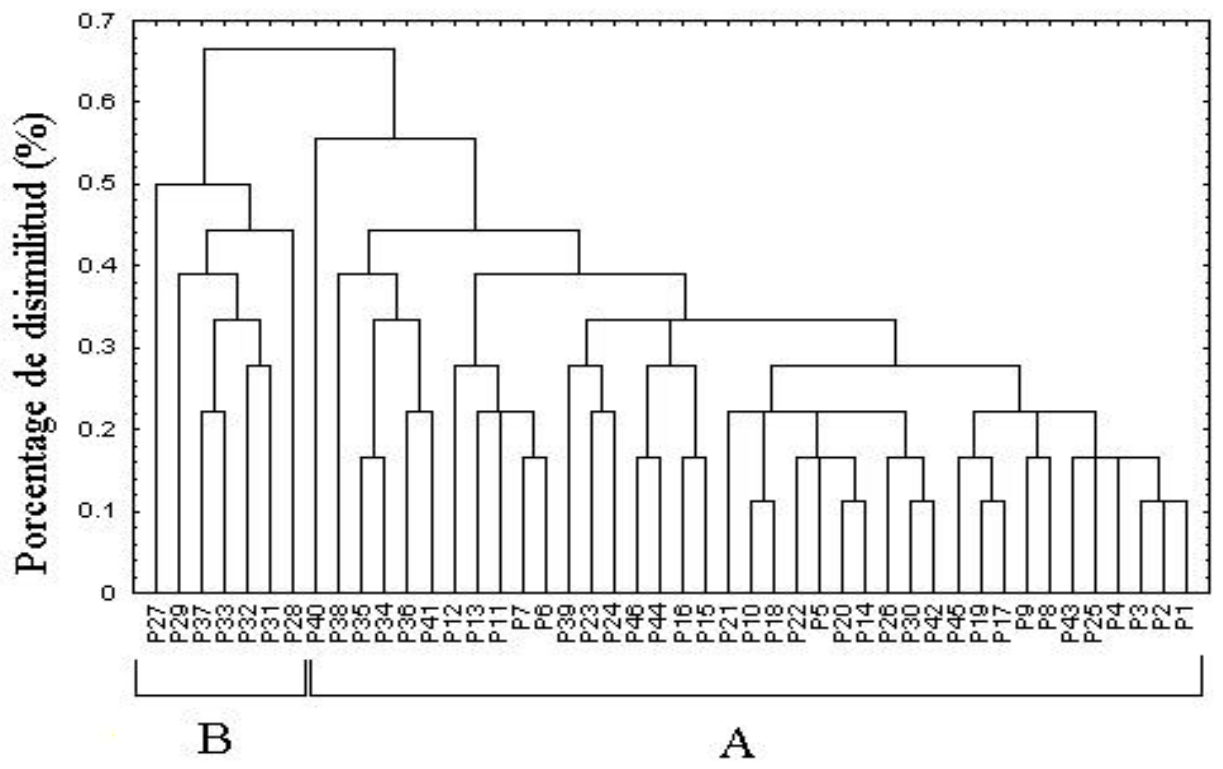

Figura 2. Dendrograma de los censos de vegetación del salar de Ascotán. Se indican las parcelas (P) y las unidades vegetacionales "A" $\mathrm{y}$ " $\mathrm{B}$ ". 


\section{DISCUSION}

FLORA ZONAL Y AZONAL

La flora azonal del salar de Ascotán resultó ser completamente diferente a la zonal de las laderas aledañas. Si bien este patrón era esperable, aún no se había cuantificado la magnitud de las diferencias para ninguno de los salares de Chile. La ausencia de especies zonales en el salar es probablemente debido a los problemas osmóticos y/o nutricionales presentados por altas concentraciones de sales (Braun-Blanquet 1979; Schat \& Scholten 1986; Shumway \& Bertness 1992). A la inversa, la baja disponibilidad de agua en las laderas circundantes podría determinar la ausencia de las especies de los humedales del salar en estos hábitats (BraunBlanquet 1979).

Las diferencias entre los espectros biológicos del salar y de la ladera sugieren que la forma de vida influye en el proceso de selección de la flora regional que se establece en cada hábitat. Las principales diferencias corresponden a la proporción de hemicriptófitas, más alta en el salar, a la de nanofanerófitas, más alta en la vegetación zonal y a la presencia de terófitas (anuales) sólo en la vegetación de las laderas. La baja proporción de hemicriptófitas en las laderas aledañas se debería a los mayores requerimientos de agua de éstas y a la escasa disponibilidad de agua y humedad en las laderas (Braun-Blanquet 1979; Arroyo etal. 1988; Danin \& Orshan 1990). La baja riqueza de nanofanerófitas en el salar, podría atribuirse a la baja tolerancia de especies nanofanerófitas en general a la salinidad y a la alta concentración de sales en su suelo (BraunBlanquet 1979). A su vez, la alta importancia de las nanofanerófitas en laderas xéricas (como las aledañas al salar) ya ha sido documentada antes por Arroyo etal. (1988) para otras localidades de clima desértico de altura del norte de Chile, no correspondiente a salar. Por otro lado, la presencia de terófitas es común en ambientes áridos (Arroyo et al. 1988) o con perturbaciones recurrentes (Tilman 1988). Si bien la presencia de estas especies en hábitats salinos es común en otras regiones biogeográficas, su presencia en ellos estaría principalmente favorecida en los sitios con menor salinidad y menor cobertura (Noe \& Zedler 2001). Luego, la ausencia de terófitas en el salar de Ascotán podría deberse al escaso espacio disponible para colonizar y a la alta salinidad de sus suelos en los espacios abiertos sin vegetación. Al respecto, Shumway \& Bertness (1992) mencionan que, en general, en los hábitats de salar y de marismas salinas, la frecuencia de perturbaciones es baja y los espacios abiertos son sólo micrositios con alta concentración de sales. Por otra parte, la mayor proporción de caméfitas en el salar respecto de la vegetación zonal, podría estar relacionada con algunas particularidades fisiológicas de especies caméfitas del salar tales como Lycium humile Phil. (Poblete et al. 1991) y Sarcocornia pulvinata (Faúndez \& Macaya 1997), ambas con hojas suculentas producto de la alta concentración de sales en su interior, la que utilizan para extraer el agua del suelo. La presencia de caméfitas con hojas suculentas ha sido también reportada para la flora de otros salares sudamericanos, tales como el de Salinas Grandes en Argentina (Ragonese 1951).

Los diferentes espectros de formas de vida del salar y vegetación aledaña confieren a ambas zonas fisonomías vegetacionales distintas. El espectro de formas de vida de la comunidad del salar de Ascotán, dominado por hemicriptófitas pulvinadas o cespitosas, es característico de los ambientes de altura, con buena disponibilidad hídrica (Raunkiaer 1934; Braun-Blanquet 1979). Este espectro le da una fisonomía de pradera, típica de los ambientes desérticos lacustres y/o salinos de tipo azonal de Chile (sensu Villagrán et al. 1983). Por otra parte, la vegetación aledaña al salar, dominada por nanofanerófitas, es característica de ambientes con estrés hídrico (Raunkiaer 1934; Braun-Blanquet 1979). La fisonomía corresponde a una estepa arbustiva o a un matorral de puna (tolar) (sensu Villagrán et al. 1982), donde predominan especies como Atriplex imbricata (Moq.) Dietr., Adesmia horrida Gillies ex Hook et Arn., Acantholippia punensis Botta y Chuquiraga atacamensis Kuntze (Teillier, obs. pers.).

Las especies que crecen en los humedales del salar se presentan también en otros salares, geográficamente distanciados, o en otras áreas con mejor disponibilidad de agua, tales como bofedales o escorrentías en quebradas del norte andino de Chile (Villagrán et al. 1982; Teillier 1998). Sin embargo, resulta interesante la presencia en el salar de Ascotán de algunas especies compartidas con regiones geográficas alejadas y sometidas a otros climas, aunque en microhábitats salinos similares, tales como Scirpus americanus Pers. y Juncus arcticus, 
presentes en las marismas salinas del sur de Chile (San Martín et al. 1992). También es relevante la presencia de géneros compartidos con estas zonas del sur de Chile tales como Triglochin, Puccinellia y Sarcocornia, pero con diferencias al nivel de especie (San Martin et al. 1992).

\section{VARIABILIDAD INTRASALAR}

La existencia de sólo dos tipos de vegetación al interior del salar, diferenciados por su composición florística, sugiere escasa variación de su ambiente abiótico. Esto, además, se refleja en que la unidad “' $\mathrm{B}$ ' se presentó sólo en un parche de vegetación, aquel aledaño a la laguna Palpana, mientras que la unidad A se encontró en ésta y en todas los demás parches. Probablemente, la zona de surgencia de agua dulce es mayor y más lejana a la laguna salina en este parche que en los otros, posibilitando una mayor humedad en una zona menos salina, y la presencia exclusiva de Festuca deserticola Phil. y Scirpus americanus Pers., ausentes en la unidad A.

Las diferencias de espectro de formas de vida entre las unidades vegetacionales del salar implican fisonomías distintas. La unidad A está dominada por caméfitas pulvinadas como Scirpus atacamensis y Sarcocornia pulvinata, ésta muy halófila y de hábito suculento no graminoide. Además, se encuentra la gramínea hemicriptófita, Puccinellia frigida, lo que le confiere a la vegetación una fisionomía de pradera baja, la que se presenta como una combinación de parches con caméfitas pulvinadas y hemicriptófitas cespitosas, bajas. En cambio, la unidad B está dominada por hemicriptófitas, principalmente cespitosas tales como Festuca deserticola y Festuca hypsophila, además de la caméfita Scirpus atacamensis de hábito pulvinado, lo que le da una fisonomía de pradera alta de tipo pajonal.

A partir de estos resultados, es posible proponer la existencia de dos unidades fitosociológicas para los humedales del salar. Una asociación de Sarcocornia pulvinata-Puccinellia frigida (A) y otra de Scirpus atacamensis-Festuca deserticola (B). La primera unidad pertenecería a la Alianza Sarcocornion pulvinatae, al Orden Anthobryetalia triandri y a la Clase Distichlio humilis-Anthobryetea triandri (Navarro 1993). Sin embargo, dado que comparte muy pocas especies con las asociaciones propuestas para esta alianza, es probable que corresponda a una nueva asociación. La segunda asociación con Scirpus atacamensis y Festuca deserticola no presenta a Sarcocornia pulvinata ni ninguna de las especies características de estas alianzas, órdenes y clases, a excepción de Distichlis humilis (Navarro 1993). Esto sugiere que esta unidad es de carácter menos halófilo y que, probablemente, se distribuye en hábitats andinos húmedos, pero con menor salinidad. Dado que se presenta en las orillas menos salinas del salar de Ascotán, la asociación podría corresponder a la clase sintaxonómica de los humedales altoandinos: Plantagini rigida-Distichietea muscoidis, al orden Calamagrostietalia jamesoni-Distichietalia muscoidis y a la alianza Wernerion pygmaeae (Navarro 1993). La presencia de especies como Puccinellia frigida, Deyeuxia eminens y Werneria pygmaea respaldaría esta propuesta. Destacamos la ausencia de Oxychloe andina, especie característica de los bofedales andinos (Navarro 1993) que estaría siendo vicariada por Scirpus atacamensis.

\section{AGRADECIMIENTOS}

Los autores agradecen a Geotécnica Consultores Ltda. y a la Compañía Minera El Abra, por las facilidades otorgadas para el estudio, al profesor C. Marticorena por su permanente disponibilidad para aclarar la taxonomía de las especies. Finalmente, agradecemos al profesor Carlos Ramírez y a dos revisores anónimos que ayudaron a mejorar sustancialmente el manuscrito.

\section{BIBLIOGRAFIA}

Armesto, J.J., P. Vidiella \& J. Gutiérrez. 1993. Plant communities of the fog-free coastal desert of Chile: plant strategies in a fluctuating environment. Revista Chilena de Historia Natural 66: 271-282.

Arroyo, M.T.K., C. Villagrán, C. Marticorena \& J.J. Armesto. 1982. Flora y relaciones biogeográficas en los Andes del norte de Chile $\left(18^{\circ}-19^{\circ} \mathrm{S}\right)$. En: $\mathrm{El}$ ambiente natural y las poblaciones humanas de los Andes del norte de Chile. Vol I: La vegetación y los vertebrados inferiores de los pisos altitudinales entre Arica y el lago Chungará (eds. A. Veloso \& E. Bustos), pp. 71-92. Rostlac, Montevideo.

Arroyo, M.T.K., F. Squeo, J.J. Armesto \& C. Villagran. 1988. Effects of aridity on plant diversity in the 
Gayana Bot. 60(2), 2003

northern Chilean Andes: results of a natural experiment. Annals of the Missouri Botanical Garden 75: 55-78.

Becerra, P. \& L. Faúndez. 2001. Vegetación del desierto interior de Quillagua, II Región. Chloris Chilensis, Año 4: 2. http://www.chlorischile.cl

Braun-Blanquet, J. 1979. Fitosociología. Bases para el estudio de las comunidades vegetales. Ediciones Blume, Madrid. 820 pp.

BRÜGgen, J. 1950. Fundamentos de geología de Chile. Instituto Geográfico Militar, Santiago, Chile. 504 pp.

Cabrera, A.1957. La vegetación de la puna Argentina. Revista de Investigaciones Agrícolas 11: 317-412.

Cody, M.1986. Structural niches in plant communities. En: Community Ecology (eds. Diamond, J. \& T. Case), pp 381-405. Harper y Row, San Francisco.

Danin, A. \& G. ORShan. 1990. The distribution of Raunkiaer life forms in Israel in relation to the environment. Journal of Vegetation Science 1: 41-48.

Di CAstri, F. \& E. Hajek. 1976. Bioclimatología de Chile. Ediciones Universidad Católica de Chile, Santiago. 129 pp.

FAúndez, L. \& J. Macaya. 1997. Presencia de Sarcocornia pulvinata (Fries) A. J. Scott (Chenopodiaceae) en Chile. Noticiario Mensual del Museo Nacional de Historia Natural Santiago 328: 3-5.

Gajardo, R. 1994. La vegetación natural de Chile. Clasificación y distribución geográfica. Editorial Universitaria. Chile. 165 pp.

Luebert, F. \& R. Gajardo. 2000. Vegetación de los Andes áridos del norte de Chile. Lazaroa 21:111-130.

Marticorena, C. \& M. Quezada. 1985. Catálogo de la flora vascular de Chile. Gayana Botánica 42: 1-157.

Messerli, B., M. Grosjean, G. Bonani, A. Bürgi, M. Geyh, K. Graf, K. Ramseyer, H. Romero, U. Schotterer, H. Schreier \& M. Vuille. 1993. Climate change and natural resource dynamics of the Atacama Altiplano during the last 18.000 years: a preliminary synthesis. Mountain Research and Development 13: 117-127.

Müller-Dombois, D. \& H. Ellenberg. 1974. Aims and methods of vegetation ecology. John: Wiley. New York. 547 pp.

Navarro, G. 1993. Vegetación de Bolivia: el Altiplano meridional. Rivasgodaya 7: 69-98.

NoE, G. \& J. ZedLer. 2001. Spatio-temporal variation of salt marsh seedling establishment in relation to the abiotic and biotic environment. Journal of Vegetation Science 12:61-74.

Ochsenius, C. 1986. La glaciación Puna durante el Wisconsin, deglaciación y máximo lacustre en la transición Wisconsin-Holoceno y refugios de megafauna postglaciales en la puna y desierto de Atacama. Revista de Geografía Norte Grande 13:29-58.

Poblete, V., L. González \& G. Montenegro. 1991. Anatomical leaf adaptations in vascular plants of a salt marsh in the Atacama Desert. Revista Chilena de Historia Natural 64: 65-75.

Ragonese, A. 1951. La vegetación de la República Argentina. Estudio fitosociológico de las Salinas Grandes. Revista de Investigaciones Agrícolas 5:1-233.

Raunkiaer, C. 1934. Plant life forms. Oxford University Press, London. 104 pp.

Rundel, P., M. Dillon \& B. Palma. 1996. Flora y vegetación del Parque Nacional Pan de Azúcar en el desierto de Atacama. Gayana Botanica 53: 295-316.

San Martín, C., D. Contreras, J. S An Martin \& C. RAMIREz. 1992. Vegetación de las marismas del centro-sur de Chile. Revista Chilena de Historia Natural 65:327-342.

Schat, H. \& M. Scholten. 1986. Effects of salinity on growth, survival and life of four short-lived pioneers from brackish dune slacks. Acta Oecologica 7:221-231.

Shumway, S. \& M. Bertness. 1992. Salt stress limitation of seedling recruitment in a saltmarsh plant community. Oecologia 92: 490-497.

Teillier, S. 1998. Flora y vegetación altoandina del área de Collahuasi - Salar de Coposa, Andes del Norte de Chile. Revista Chilena de Historia Natural 71: 313-329.

Teillier, S. 2000. Flora vascular del salar de Ascotán, Región de Antofagasta (II). Chile. Chloris Chilensis, Año 3:1. http://www.chlorischile.cl

Tilman, D. 1988. Plant strategies and the dynamics and structure of plant communities. Monographs in population biology. Princeton University Press, New Jersey, USA. 360 pp.

VILA, T. 1975. Geología de los depósitos salinos andinos, provincia de Antofagasta, Chile. Revista de Geología de Chile 2: 41-55.

Villagrán, C., M.T.K. Arroyo \& J.J. Armesto. 1982. Vegetación de una transecta altitudinal en los Andes del Norte de Chile $\left(18^{\circ}-19^{\circ} \mathrm{S}\right)$. En: El ambiente natural y las poblaciones humanas de los Andes del Norte Grande de Chile. Vol I: La vegetación y los vertebrados inferiores de los pisos altitudinales entre Arica y el lago Chungará (eds. A. Veloso \& E. Bustos), pp. 13-69. Rostlac, Montevideo.

Villagrán, C., M.T.K. Arroyo \& C. Marticorena. 1983. Efectos de la desertización en la distribución de la flora andina de Chile. Revista Chilena de Historia Natural 56: 137-157. 